for the semiconductor properties of these compounds are shown in the energy pulse ( $\hbar k$ ) diagram (Fig. 3). Although the valence band is divided into several subbands it is still possible to operate in the first approximation with a centrally symmetric parabolic band. In the conduction band, however, secondary minima appear at or near the boundary of the Brillouin zone in the $/ 100 /$ or $/ 111 /$ direction in addition to the central minimum. Completely different semiconductor properties result, depending on the energy level of these minima. If the central minimum has the lowest energy level, extremely high electron mobility will be observed. Electrons brought into this minimum by external excitation will cross directly from the conduction to the valence band (cf. Fig. 3, (a)) and light emission will occur. If the secondary minima have the lowest level, the electron mobility will be lower. Electrons may only cross indirectly from these minima to the valence band (cf. Fig. 3, (b)) and long carrier lifetimes will be possible. Finally it should be added that if charge carriers of high mobility are forced by an applied electric field from the central minimum to a satellite minimum (cf. Fig. 3, (c)), negative resistances will appear (Gunn effect) which, for instance, have assumed importance for $\mathrm{GaAs}$ or InP microwave generators. Except for this Gunn effect the properties of III-V compounds which are of significance as compared to those of germanium and silicon were already experimentally demonstrated by Welker and coworkers and the resulting possibilities with regard to the large range of variation of the band gap recognized. At first, however, only galvanomagnetic devices, which utilize the high mobility of the majority carriers, made any headway.

The technology for more sensitive effects still had to be developed. It was here that past experience with silicon, especially in connection with the

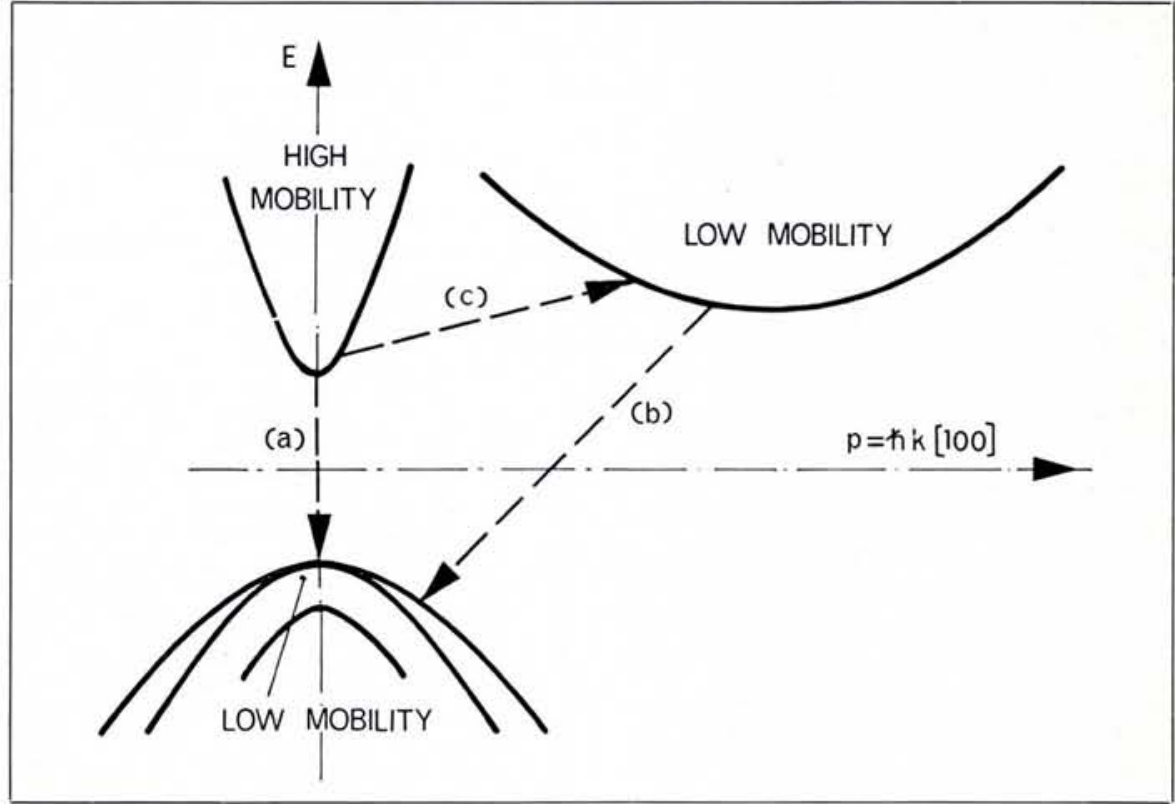

Fig. 3 Band structure diagram

deposition of ultrapure and perfect epitaxial layers, proved useful. Yet another impetus from the silicon sector proved of decisive importance for optoelectronics, the currently largest branch of III-V semiconductor technology: a demand for optoelectronic devices capable of operating with the same low voltages as integrated circuits. Thus the advent of GaAs, Ga (AsP) and $\mathrm{GaP}$ light-emitting diodes led to the creation of a market whose worldwide sales have already surpassed the $\$ 200$ Mill. mark. Not only would the simple LED with its broad spectrum extending from near infrared to light green be inconceivable without III-V compounds, but even the far more complex laser diodes built of GaAs and (GaAl)As layers could never have been realized without them. Designed to emit and amplify finely focused coherent light signals, this laser diode is ideally suitable for communications transmission. Like GaAs LEDs with their incoherent light it represents, by allowing easy modulation up to about the $\mathrm{GHz}$ region, a key device for future communication systems in which guided light waves will be transmitted along glass fibres. The future market can of course only begin to develop after the overall system has been brought to maturity.

Like that for the laser diode, the market for the large variety of microwave devices made of III-V compounds, GaAs Schottky-barrier diodes, GaAs tunnel diodes, GaAs FETs as well as GaAs and InP Gunn-effect elements is still in its infancy. These devices will only attract real interest after a sufficient demand has developed for frequencies in the region, of $10 \mathrm{GHz}$ and higher, where silicon approaches a limit set by its lower electron mobility.

Although this short review could only treat the principal aspects of III-V semiconductors, these alone already demonstrate what a vast and far-reaching area of science and technology has been opened up. Its importance has been adequately highlighted by the honorable award to the man who made this possible.

\title{
3rd General Assembly of EPS Members
}

The Third General Assembly of EPS members took place in Bucharest on Wednesday evening 10 September 1975. The President, H.B.G. Casimir, presented his report on the state of the society, stressing the difficult period passed by the Society due to the weak financial situation. He mentioned the personnel changes and further measures which have taken place at the Secretariat since Wiesbaden and he considered that the Society's acti- vities should be able to make further progress, backed by an organization that is in a healthy state. The President then gave a review of the most recent developments. Europhysics News was now being published according to recommendations made by an Advisory Committee and a new Advisory Committee on Physics Education was to have its constitutional meeting the following day.

The Secretary, M. Guenin, gave some details on administrative matters. The main problem was to find a way to improve the financial situation. Of the two possibilities, reducing expenditure or increasing the income, the first alternative had been applied to such an extent that new cuts would mean reducing the staff at the Secretariat to little more than zero. The Secretary announced the new publication Europhysics Conference Abstracts. He expressed the hope that 
although the first objective of this project was to give more information to the physics community, it would also develop into a source of income in the long run.

The Treasurer, O.S. Heavens, showed through a multitude of figures, that the financial situation although still marked by a considerable accumulated deficit, was moving uphill with a small but regular profit on operations in the current and coming years. Despite the heavy cut in expenditure the unit fee had to be increased twice since Wiesbaden. The first increase in 1974 was necessary to adapt the income to a certain extent to inflation, the second decided at the Council meeting of 8 September 1975 , was mainly recommended to avoid a similar weak financial situation within a few years.

The Chairman of the Advisory Committee on Conferences, P. Radvanyi, reported on the results obtained in the coordination of meetings. More effort should be put into the cooperation with conferences organized outside the regions covered by EPS.

The first President, G. Bernardini, started the general discussion with some kind words on the organization of the third General Conference and the zeal of the local authorities. He also expressed the wish that special attention be given to the publication of the conference.

One firm suggestion made, was to organize a general conference every two years instead of the present three. The Secretary answered that this suggestion would be studied by the Executive Committee but that it was already too late to plan for a conference in 1977. The next general conference is scheduled for September 1978 either in one of the Scandinavian countries or in York (U.K.). The President made some remarks on the consequences to be considered in organizing the general conference at shorter intervals: a permanent Programme Committee would probably have to be formed to prevent the general conference becoming too specialized; also the financial aspects would have to be studied carefully.

Other suggestions concerning the general conference were briefly discussed, an enlarged use of mass media, the possibility of opening some sessions to a broader public, the preparation of extensive summaries of the conference for publication.

G. Thomas Geneva

NITWBOCKS

\section{In-Beam Gamma-Ray Spectroscopy}

by H. MORINAGA and T. YAMAZAKI.

1976 about 550 pages

US\$ 62.50 / Dfl. 150.00

This book, in a sense supplementing Siegbahn's "Alpha-, Beta-, and Gamma-Ray Spectroscopy", opens with accounts of various experimental and theoretical techniques relevant to inbeam gamma ray measurements. Various types of spectroscopy are then described, namely spectroscopy with simple nuclear reactions and with continuum reactions; measurements of life-times and nuclear moments in beam, neutron capture gamma rays, and photoexcitations are dealt with, together with a discussion on electromagnetic properties of the nucleus revealed by in-beam gamma ray spectoscopy.

CONTENTS: Progress of detection techniques. Principles of gamma ray spectroscopy. Electromagnetic properties of nuclei. Nuclear reaction mechanisms. Gamma rays in low energy nuclear reactions. Gamma rays in direct nuclear reactions. Gamma rays in compound continuum reactions. Delayed gamma rays. Perturbed angular distributions and nuclear moments. Nuclear photoeffects. Gamma rays from neutron capture.

\section{The Electromagnetic Interaction in Nuclear Spectroscopy}

edited by W. D. HAMILTON.

$1975 \times \mathbf{x}+925$ pages

US $\$ 108.50 /$ Dfl. 260.00

The papers in this multi-author work combine to form a comprehensive and unified treatment of the basic theoretical and experimental methods of nuclear spectroscopy. Part of the book deals with semiconductor detectors, as research in this area has been particularly fruitful. The many factors which must be taken into account, if these detectors are to achieve their full potential, are extensively discussed.

CONTENTS: Emission and absorption of electromagnetic radiation ( $K$. Alder and R. M. Steffen). Emission of gamma radiation and nuclear structure $(K$. Alder and R. M. Stetfen). Collective and microscopic model predictions of electromagnetic moments (K. Kumar). Nuclear shapes and phase transitions (K. Kumar). Transition probabilities
(K. E. G. Lobner). Delayed coincidence timing methods (K. E. G. Lobner). Coulomb excitation (J. O. Newton). Nuclear resonance fluorescence (S. J Skorka). Doppler shift timing methods (K. W. Allen). The theory of internal conversion (K. Alder). Experimental techniques of conversion coefficient measurements (J.H. Hamilton). Angular distribution and correlation of gamma rays, theoretical basis (R. M. Steffen and $K$. Alder). Extranuclear perturbations of angular distributions and correlations (R.M. Steffen and K.Alder). Angular distribution and correlation measurements using radioactive sources (W. D. Hamilton). Angular distribution and correlation of gamma ray (P. J. Twin). Measurement of electric and magnetic moments of excited states ( $E$. Bodenstedt). Gamma-ray energy and intensity measurements with a curved crystal spectrometer (J. J. Reidy). Gamma-ray energy and intensity measurements with $\mathrm{Ge}(\mathrm{Li})$ spectrometers (R.G. Helmer, J.E. Cline and R. C. Greenwood). Subject index.

\section{Atomic and Molecular Physics and the Interstellar Matter}

Proceedings of the XXVI Session of the Les Houches Summer School in Theoretical Physics, July-August, 1974

edited by ROGER BALIAN, PIERRE ENCRENAZ and JAMES LEQUEUX.

1975 xxiv +634 pages

US $\$ 66.75 /$ Dfl. 160.00

The Les Houches Summer School in Theoretical Physics 1974, was devoted to the physics of interstellar matter with special emphasis on atomic and molecular processes. The eight-week duration of the conference made it possible to examine in depth a broad range of topics.

CONTENTS: Atomic physics (H. Nussbaumer). Molecular spectroscopy and collisional excitation (S. Green). Atomic collision theory (D. Flower). Atomatom collision processes in the interstellar medium (R. McCarroll). Formation and destruction of interstellar molecules (W. D. Watson). Physics of fully ionized regions (D. Flower). Observations and physics of dense neutral clouds (A. A. Penzias). Interstellar masers ( $P$. Goldreich). Interstellar grains (E. M. Greenberg). Heating and ionization of the interstellar medium: star formation (G. B. Field). Dynamics of interstellar matter (F. D. Kahn). Nucleosythesis and the origin of galactic cosmic rays ( $H$. Reeves). Seminars given during the school 